\title{
Sigambra sundarbanensis sp. nov. (Annelida, Pilargidae) from the Indian sector of Sundarbans Estuarine System, with remarks on parapodial glands
}

\author{
Moumita BHOWMIK ${ }^{1}$, Priya GHOSHAL ${ }^{2}$, Sergio I. SALAZAR-VALLEJO ${ }^{3} \&$ \\ Sumit MANDAL ${ }^{4, *}$ \\ ${ }^{1,2,4}$ Marine Ecology Laboratory, Department of Life Sciences, Presidency University, 86/1, \\ College Street, Kolkata 700073, India. \\ ${ }^{3}$ Departamento de Sistemática y Ecología Acuática, El Colegio de la Frontera Sur, \\ Chetumal, Q. Roo, Mexico. \\ "Corresponding author: sumit.dbs@presiuniv.ac.in \\ ${ }^{1}$ Email: moumitabhowmik20@gmail.com \\ 2Email: ghoshalpriya97@gmail.com \\ ${ }^{3}$ Email: savs551216@hotmail.com

\footnotetext{
${ }^{1}$ urn:1sid:zoobank.org:author:7A1A38D9-E8C6-4401-A051-BA1BB02D6662

${ }^{2}$ urn:1sid:zoobank.org:author:908F1B61-37B1-44C0-BD5B-247987788F4C

${ }^{3}$ urn:1sid:zoobank.org:author:7D3C2D7E-5D08-45F7-A1E4-84A1563EA14F

${ }^{4}$ urn:lsid:zoobank.org:author:6662EECE-73E6-426F-B596-98632AB4DB9A
}

\begin{abstract}
A new pilargid species, Sigambra sundarbanensis sp. nov., is described from the rivers Matla and Thakuran, in the central Indian sector of the Sundarbans Estuarine System. This species is characterized by several characters such as the starting position of the notopodial hooks, the length of the median antenna and the variation in number of the neuropodial chaetae. These characters distinguish the new species from its congeners. Some parapodial glands have been found in individuals of this species. The new species closely resembles Sigambra parva (Day, 1963). Additionally, an updated key of genus Sigambra is provided, along with a table indicating their morphological variations and a global map showing their type localities.
\end{abstract}

Keywords. Polychaeta, Pilargidae, parapodial glands, estuaries, Bay of Bengal.

Bhowmik M., Ghoshal P., Salazar-Vallejo S.I. \& Mandal S. 2021. Sigambra sundarbanensis sp. nov. (Annelida, Pilargidae) from the Indian sector of Sundarbans Estuarine System, with remarks on parapodial glands. European Journal of Taxonomy 744: 49-66. https://doi.org/10.5852/ejt.2021.744.1301

\section{Introduction}

Pilargids are uncommon nereidiform marine annelids; most are free-living, and many are motile burrowers. They are ubiquitous in sediments at various depths in estuarine or oceanic realms, most preferably in coarse to mixed substratum (Jumars et al. 2015). They are considered carnivores and 
omnivores, but perhaps they intake detritus and microalgae (Day 1967; Dean 1998). The body is often depressed and ribbon-shaped. The integument is smooth or papillated. The pharynx is bulbous, usually papillated, it has rigid structures, sometimes with circlets of marginal papillae. The first dorsal cirri are often longer than the following. Notopodia are always reduced with embedded notoacicula, with or without additional capillary chaetae. Globally, Pilargidae de Saint-Joseph, 1899 comprises about 112 accepted species, under 12 genera (WoRMS 2020). This family has been reviewed several times by Hartman (1947), Pettibone (1966), Salazar-Vallejo (1986) and Licher \& Westheide (1997). The phylogenetic affinities of Pilargidae have been questioned over a long time; based on morphological and behavioural similarities. Licher \& Westheide (1994) considered the pilargids as a derived group within hesionids. However, further investigations by Pleijel \& Dahlgren (1998) and Dahlgren et al. (2000) rejected the previous hypothesis of inclusion of pilargids within Hesionidae (Grube, 1850), and recognized them as non-overlapping families.

Sigambra Müller, 1858 is one of the most specious genera in Pilargidae and contains about 27 accepted species globally (Salazar-Vallejo et al. 2019). Müller (1858) proposed eight new genera from Santa Catarina Island (Brazil), including Sigambra. Later on, Hartman (1947) described some species, but regarded them as Ancistrosyllis McIntosh, 1879. A few species of Ancistrosyllis were reinstated under Sigambra. Sigambra has dorsal hooks above the dorsal cirri, resembling Ancistrosyllis, but they differ by the size of body appendages, antennae, tentacular cirri and body papillation (Salazar-Vallejo \& Rizzo 2009).

In India, taxonomic reports of only two species of Sigambra have thus far been published: $S$. constricta Southern, 1921, later recorded by Misra (1995) from Chilka Lake and Hooghly-Matla Estuary, respectively, and S. tentaculata (Treadwell, 1941) from the Indian southwest coast (Achari 1975). Even though other species of this genus, such as $S$. parva (Day, 1963) and S. bassi (Hartman, 1947), have also been reported in macrobenthic community studies from various estuaries, coasts and tidal creeks, no further taxonomic information was provided. Due to a low number of surveys, our knowledge concerning the taxonomy and ecology of polychaetes from the central sector of the Sundarbans Estuarine System (SES) is minimal (see Mandal \& Deb 2018). In this contribution, we describe a new species of Sigambra from the rivers Matla and Thakuran in the SES delta. Furthermore, we report on some glandlike structures in parapodial spaces, which have not been reported from any species of this genus.

\section{Material and methods}

\section{Study site}

Sundarbans Estuarine System (SES) is the largest monsoonal micro-tidal delta comprising hundreds of estuaries located alongside the Indian coast. It is both a World Heritage Site declared by UNESCO in 1997 and a RAMSAR site since 2019 (http://wiienvis.nic.in/Database/ramsar_wetland_sites_8224.aspx). The rivers Matla and Thakuran are two major rivers in the system. Because they are in the central zone, these rivers have been disconnected from freshwater supply and lost their estuarine pattern due to high siltation and neotectonic activity causing tilting of the Eastern Bengal Basin in recent years (Stanley \& Hait 2000; Manna et al. 2010; Raha et al. 2012). Samples of macrobenthos, water and sediment were collected during SES biological surveys under an ongoing project (stations were marked according to that study), in January 2019, August 2019 and December 2019 from both rivers (Fig. 1).

\section{Treatment of specimens}

Material was collected in triplicate using a Van Veen grab $\left(0.04 \mathrm{~m}^{2}\right)$. The samples were separately washed in situ through a $0.5 \mathrm{~mm}$ sieve. Material retained in the sieve was allowed to relax in a solution of $7 \% \mathrm{MgCl}_{2}$ in sea water and immediately fixed in a solution of $4 \%$ buffered formalin in sea water (for a few samples from January 2019, Rose Bengal was added during fixation). The samples were 

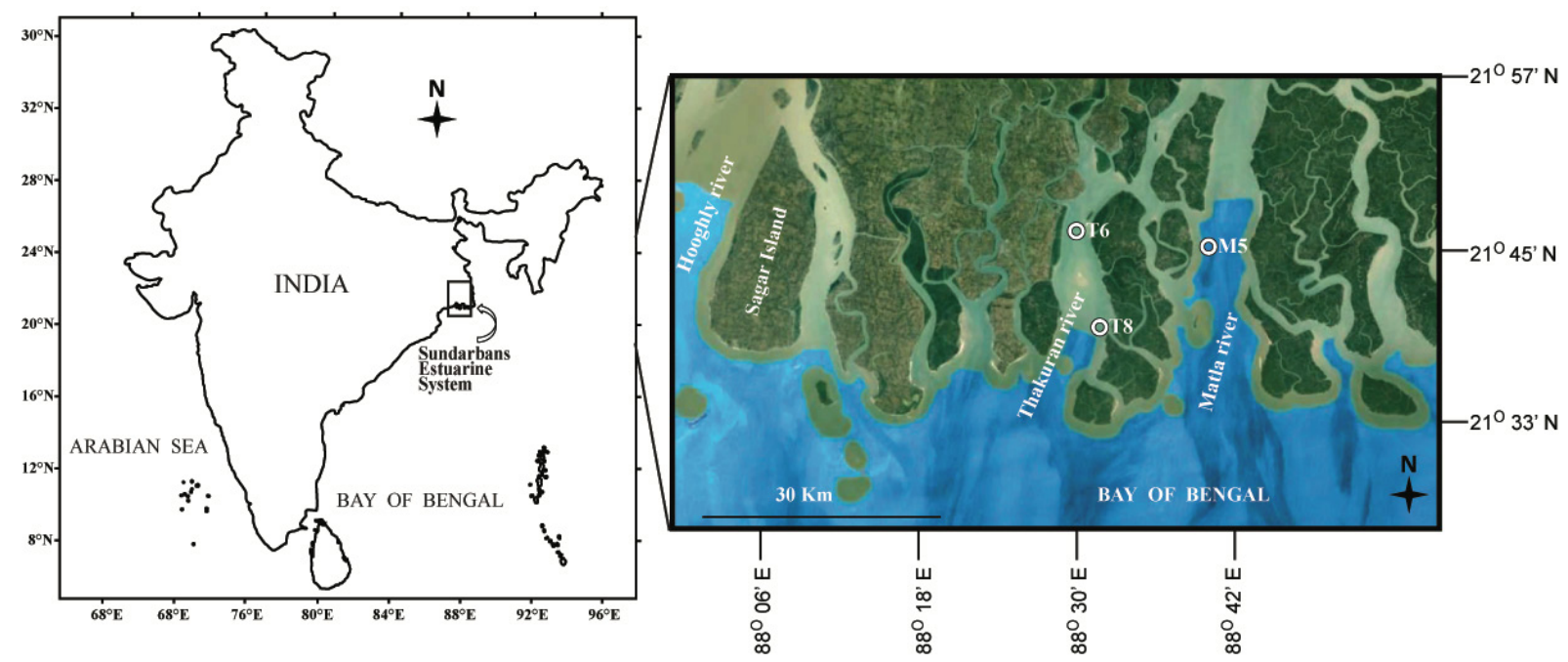

Fig. 1. Study area showing the sampling stations of our ongoing sampling program at SES, indicated by numbers, where $\mathrm{M}=$ Matla River and $\mathrm{T}=$ Thakuran River.

transported to the laboratory and later sorted and preserved in $70 \%$ ethanol. For better observation of the parapodial structures, and to get a clear visualization of the starting position of the notopodial hooks, the marginal papillae and the oocytes, specimens were mounted in a 1:1 solution of glycerin and $70 \%$ ethanol. Methyl green staining is often used to examine internal structures such as parapodial glands, as most glands contain phospholipids and the stain is more intensely fixed upon them. Our specimens were first immersed in an oversaturated methyl green solution in 70\% ethanol (as samples were preserved in the same concentration of ethanol) for about $1 \mathrm{~min}$; then, they were briefly set on tissue paper and further rinsed in clean ethanol. Samples were scanned and photographed through stereo (Olympus SZX7) and compound (Nikon ECLIPSE Ci) microscopes. For environmental parameters (temperature, $\mathrm{pH}$, salinity, sediment texture and organic content), bottom waters and sediment samples were collected on-board and analyzed using standard protocols (El Wakeel \& Riley 1957; Buchanan 1984; Grasshoff et al. 1999). The holotype and all paratypes were deposited at the Zoology Museum, Department of Life Sciences, Presidency University, Kolkata, India (PUZ).

\section{Results}

\section{Description of new species}

Class Polychaeta Grube, 1850

Order Phyllodocida Dales, 1962

Suborder Nereidiformia Glasby, 1993

Family Pilargidae de Saint-Joseph, 1899

Subfamily Pilarginae de Saint-Joseph, 1899

Genus Sigambra Müller, 1858

Sigambra sundarbanensis sp. nov. urn:1sid:zoobank.org:act:D315C406-6F83-413C-BFCA-E00A8D83070C

Figs 2-5; Table 2

\section{Diagnosis}

A species of Sigambra with median antenna reaching up to chaetigers 3-4, 2-3 times as long as lateral antennae; tentacular segment 3-4 times as wide as long. Pharynx with 14 prismatic projected lobes. 
Dorsal cirri larger than ventral ones, largest in chaetiger 1 . Ventral cirri absent in chaetiger 2 . Notopodial hooks start in chaetiger 8, accompanied by notoacicula; neuropodia with various types of capillary chaetae. Parapodial spaces with glandular, tubular structures.

\section{Etymology}

The type locality (river Thakuran) is a tidal estuarine river of the Sundarbans Estuarine System. The epithet of this new species refers to the entire estuarine system, i.e., Indian Sundarbans.

\section{Type material}

\section{Holotype}

INDIA • complete spec.; river Thakuran, stn T8; 2139'3.73" N, 88³0'25.17" E; depth 26 m; Aug. 2019; Moumita Bhowmik and Sumit Mandal leg.; in sediment; PUZ 501.

\section{Paratypes}

INDIA • 4 complete specs; river Thakuran, stn T6; $21^{\circ} 45^{\prime} 35.90^{\prime \prime}$ N, 88 $88^{\circ} 9^{\prime} 8.53^{\prime \prime}$ E; depth 10 m; Aug. 2019; Moumita Bhowmik and Sumit Mandal leg.; in sediment; PUZ 502 to PUZ 505 - 3 complete specs; river Thakuran, stn T8; 2139'3.73" N, 88 30'25.17" E; depth 26 m; Aug. 2019; Moumita Bhowmik and Sumit Mandal leg.; in sediment; PUZ 506 to PUZ 508 - 6 complete specs; river Thakuran, stn T8; 2139'3.73" N, 88³0'25.17" E; depth 26 m; Dec. 2019; Moumita Bhowmik and Sumit Mandal leg.; in sediment; PUZ 514 to PUZ 519 - 2 incomplete specs; river Matla, stn M5; $21^{\circ} 45^{\prime} 18.20^{\prime \prime} \mathrm{N}$, 88 38'25.20" E; depth $11 \mathrm{~m}$; Jan. 2019; Moumita Bhowmik and Sumit Mandal leg.; in sediment; PUZ 490 to PUZ 491.

\section{Sampling site and type locality}

Various environmental factors that characterize the sampling sites are in Table 1. Bottom water salinity ranged from 17.0 in August to 23.42 in January 2019. Sediment temperature was found to be at its maximum in August 2019. Organic enrichment in sediment was moderate, ranging from 0.78 to $1.78 \%$. In terms of granulometry, the study sites are mostly silty with comparatively finer and coarser particles that vary seasonally. The lowest proportion of clay was represented in the soil texture during the monsoon $(0.15-0.35 \%)$. The sediment texture of the type locality was characterized by a high silt percentage and a lower sand percentage that further decreased in the post-monsoon season (Dec. 2019). Bottom water salinity level varied from 17 to 21 (Table 1). Morphological and morphometric data are in Table 2 and the comparison of the new species with all other accepted species of Sigambra is in Table 3.

The holotype of Sigambra sundarbanensis sp. nov. was collected from the river Thakuran (station T8) and paratypes were collected from both the rivers Thakuran and Matla in January 2019, August 2019 and December 2019. A morphometric analysis was performed for all the collected specimens. Moreover, a global map (Fig. 2) has been presented for all the accepted species of Sigambra based on their type locations.

\section{Description}

\section{Holotype (PUZ 501)}

MeAsurements. Complete, $5.63 \mathrm{~mm}$ long, $0.32 \mathrm{~mm}$ wide at chaetiger $8-9$ (average width $0.28 \mathrm{~mm}$ ), 64 chaetigers (Fig. 3A).

BoDy. Obconic, sub cylindrical along anterior end, depressed thereafter.

Prostomium. Blunt, bilobed, three times as wide as long. Palps biarticulated directed ventrally; palpophores large, palpostyles small. Pharynx exposed with 14 prismatic marginal papillae, tips distinct 
Table 1. Environmental parameters of studied stations.

\begin{tabular}{lcccc}
\hline Sampling period & Jan. 2019 & \multicolumn{2}{c}{ Aug. 2019 } & Dec. 2019 \\
\hline Station name & M5 & T6 & T8 & T8 \\
Depth $(\mathrm{m})$ & 11 & 10 & 26 & 26 \\
Bottom water salinity (PSU) & 23.42 & 19 & 17 & 21 \\
Sediment temperature $\left({ }^{\circ} \mathrm{C}\right)$ & 21 & 31 & 31.5 & 26 \\
Organic matter $(\%)$ & 1.78 & 0.9 & 0.78 & 1.33 \\
Sand $(\%)$ & 2.78 & 35.95 & 33.23 & 6.23 \\
Silt $(\%)$ & 83.82 & 63.7 & 66.62 & 77.97 \\
Clay $(\%)$ & 13.4 & 0.35 & 0.15 & 15.8 \\
\hline
\end{tabular}

(Fig. 3C). Antennae cirriform, lateral antennae subdistally located, smaller than median one (Fig. 3B). Median antenna 2.3 times as long as laterals, reaching up to chaetiger 4.

Tentacles. Tentacular segment 3-4 times as wide as long; two pairs of tentacular cirri, dorsal tentacular cirri slightly larger than ventral ones.

CIRRI. Parapodial cirri triangular, tapered, foliose, longer than wide. Dorsal cirri longer than ventral cirri throughout, largest in chaetiger 1, reaching up to chaetiger 5 (Fig. 3D). Chaetiger 2 with smallest dorsal cirri, without ventral cirri. Parapodia with reduced notopodia and well developed neuropodia.

NoтороDiA. Include distally curved dorsal hooks from chaetiger 8 (Fig. 3D), head of hook not exposed outside body wall to chaetiger 22, fully exposed from chaetiger 23, continued along body (Fig. 3E) up to last 2 pre-pygidial chaetigers (Fig. 3G). From chaetiger 8 onwards, hooks accompanied with acicula (Fig. 5A-B). Neurochaetae include 2-4 short wide pectinate chaetae with variable number of spinulose or serrated capillaries (Figs 3F, 5A).

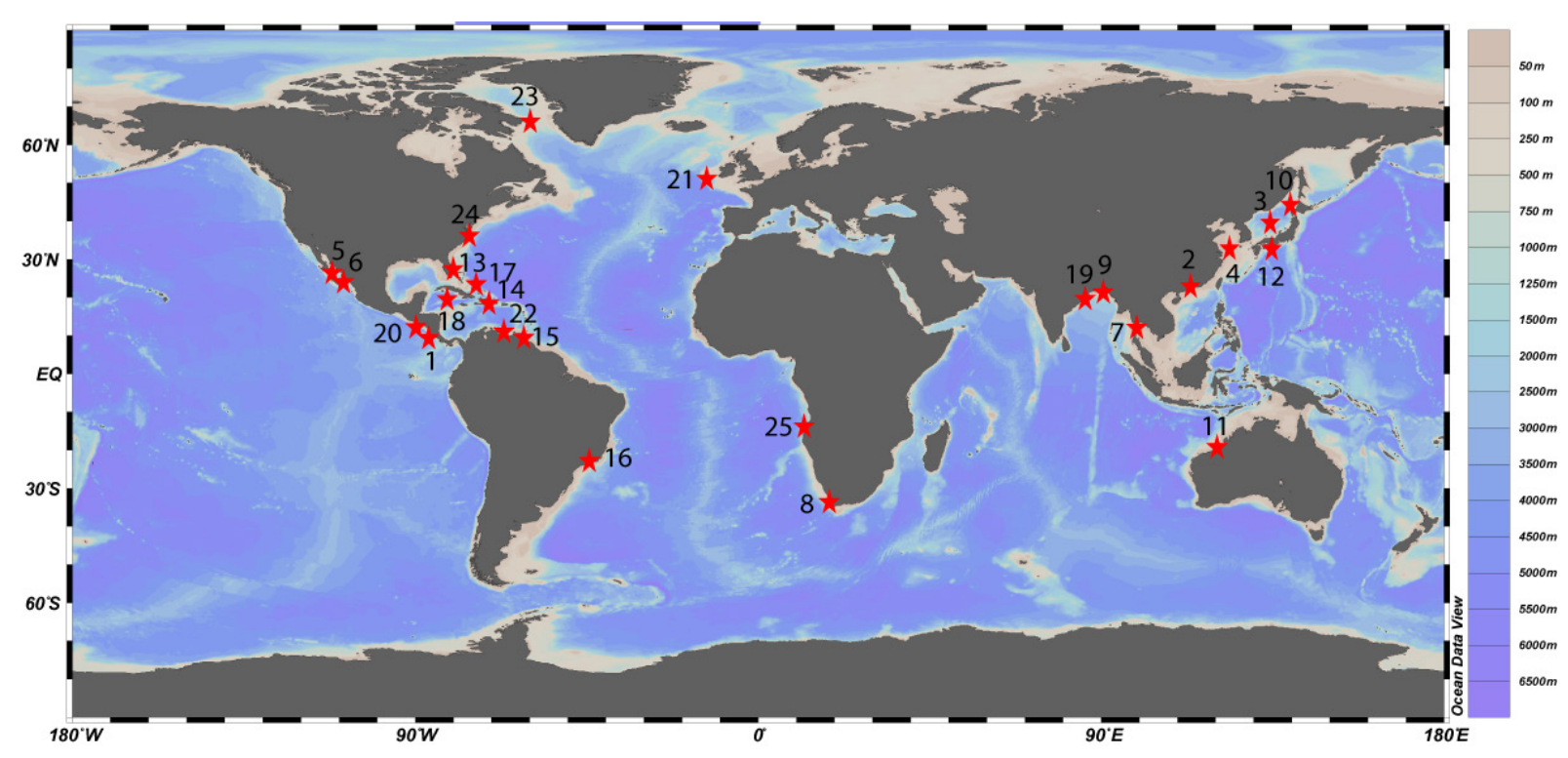

Fig. 2. Type localities of the accepted species of Sigambra Müller, 1858, names of the species are denoted with the serial numbers from Table 3. 
Table 2. Morphometric data of holotype and paratypes of Sigambra sundarbanensis sp. nov.

\begin{tabular}{lcc}
\hline & Holotype & Paratypes $(\mathbf{n}=\mathbf{1 5})$ \\
\hline Length $(\mathrm{mm})$ & 5.63 & $2.18-8.91 \mathrm{~mm}(5.09 \pm 2.29)$ \\
Width $(\mathrm{mm})$ & 0.28 & $0.08-0.41(0.15 \pm 0.08)$ \\
No. of chaetiger & 64 & $32-79$ \\
No. of marginal papillae & 14 & 14 \\
Length of median antenna $(\mathrm{mm})$ & 0.36 & $0.2-0.57(0.36 \pm 0.11)$ \\
Right lateral antenna $(\mathrm{mm})$ & 0.172 & $0.06-0.35(0.18 \pm 0.07)$ \\
Left lateral antenna $(\mathrm{mm})$ & 0.15 & $0.08-0.38(0.19 \pm 0.08)$ \\
Tentacular segment $(\mathrm{L}: \mathrm{W})$ & $1: 4$ & $1: 4$ \\
Tentacular cirri (right) $(\mathrm{mm})$ & 0.18 & $0.09-0.22(0.17 \pm 0.04)$ \\
Tentacular cirri $($ left $)(\mathrm{mm})$ & 0.18 & $0.09-0.26(0.17 \pm 0.04)$ \\
Dorsal cirri:Ventral cirri & Dorsal $>$ Ventral $(0.87$ times as long $)$ & Dorsal $>$ Ventral $(0.5-0.9$ times as long $)$ \\
First appearance of notopodial hook & Chaetiger 8 & Chaetiger 8 \\
Size of notopodial hook $(\mathrm{mm})$ & 0.13 & $0.12-0.18(0.14 \pm 0.01)$ \\
Diameter of oocyte $(\mu \mathrm{m})$ & $\mathrm{NA}$ & $12-36(23.33 \pm 6.90)$ \\
Parapodial gland length $(\mu \mathrm{m})$ & $19-50(29 \pm 16.38)$ & $14-74(43.88 \pm 17.69)$ \\
Parapodial gland width $(\mu \mathrm{m})$ & $8-11(9 \pm 1.41)$ & $8-18(2.38 \pm 10.23)$ \\
\hline
\end{tabular}

GLANDS. Parapodial glands starting from chaetiger 5, developed gradually up to chaetiger 60 . Each gland with 2-6 large tubular cells, varying in shape and size (Fig. 4B, 5D). These tubular structures converge ventrally from wide base of coelomic ramus. Tubular structures rudimentary (L: $19 \mu \mathrm{m}, \mathrm{W}: 11 \mu \mathrm{m})$ or fully developed (L: $50 \mu \mathrm{m}, \mathrm{W}: 8 \mu \mathrm{m}$ ); inner features unknown.

PyGidium. Laterally expanded with 2 ventral cirri, as long as 3-4 median chaetigers (Fig. 3G).

Oocytes. Not seen.

\section{Paratypes}

A total of 13 complete and 2 incomplete paratypes show a minor characteristic variation. They were $2.18-8.91 \mathrm{~mm}$ long $(5.09 \pm 2.29 \mathrm{~mm}), 0.08-0.41 \mathrm{~mm}$ wide $(0.15 \pm 0.08 \mathrm{~mm})$; median antennae were $0.2-0.57 \mathrm{~mm}$ long $(0.36 \pm 0.11 \mathrm{~mm})$ reaching up to chaetigers 3-4. Oocytes (Figs 4A, 5C) 12-36 $\mu \mathrm{m}$ in diameter $(23.33 \pm 6.90 \mu \mathrm{m})$. Glandular structures in parapodial spaces have been found in most paratypes, they were $14-74 \mu \mathrm{m}$ long $(43.88 \pm 17.69 \mu \mathrm{m})$ (Table 2). Large tubular glandular cells in chaetigers 47-49 of paratype PUZ 506 are shown in Fig. 4C-D. In other parapodia (chaetigers 12 and 13), tubular cells invade into coelomic space (Fig. 4E-F).

\section{Remarks}

Following the redescription of S. parva by Moreira \& Parapar (2002), it can be stated that S. sundarbanensis sp. nov. resembles $S$. parva Day, 1963. They have similar characteristics, such as median antenna longer than lateral ones, reaching chaetigers 3-4, and pharynx with 14 marginal papillae. However, they differ in several features, the most notable ones being the starting point of the dorsal hooks and the absence of capillary chaetae in the notopodia. In S. sundarbanensis sp. nov., the first appearance of dorsal hooks from chaetiger 8 remains constant in all 16 specimens, irrespective of specimen size. The hooks are accompanied by a single acicula, and the last two chaetigers are hookless. The notopodia are devoid of any capillary chaetae, neuropodia with 2-4 short pectinate chaetae with a variable number of spinulose or serrated capillaries, and the relative size of the median antenna is 2.3 times as long as the lateral ones. In comparison with $S$. parva, the median antenna is 1.5 times as long as the lateral ones, the notopodial hook starts from chaetigers $4-5$ and is accompanied by single capillary chaetae in 

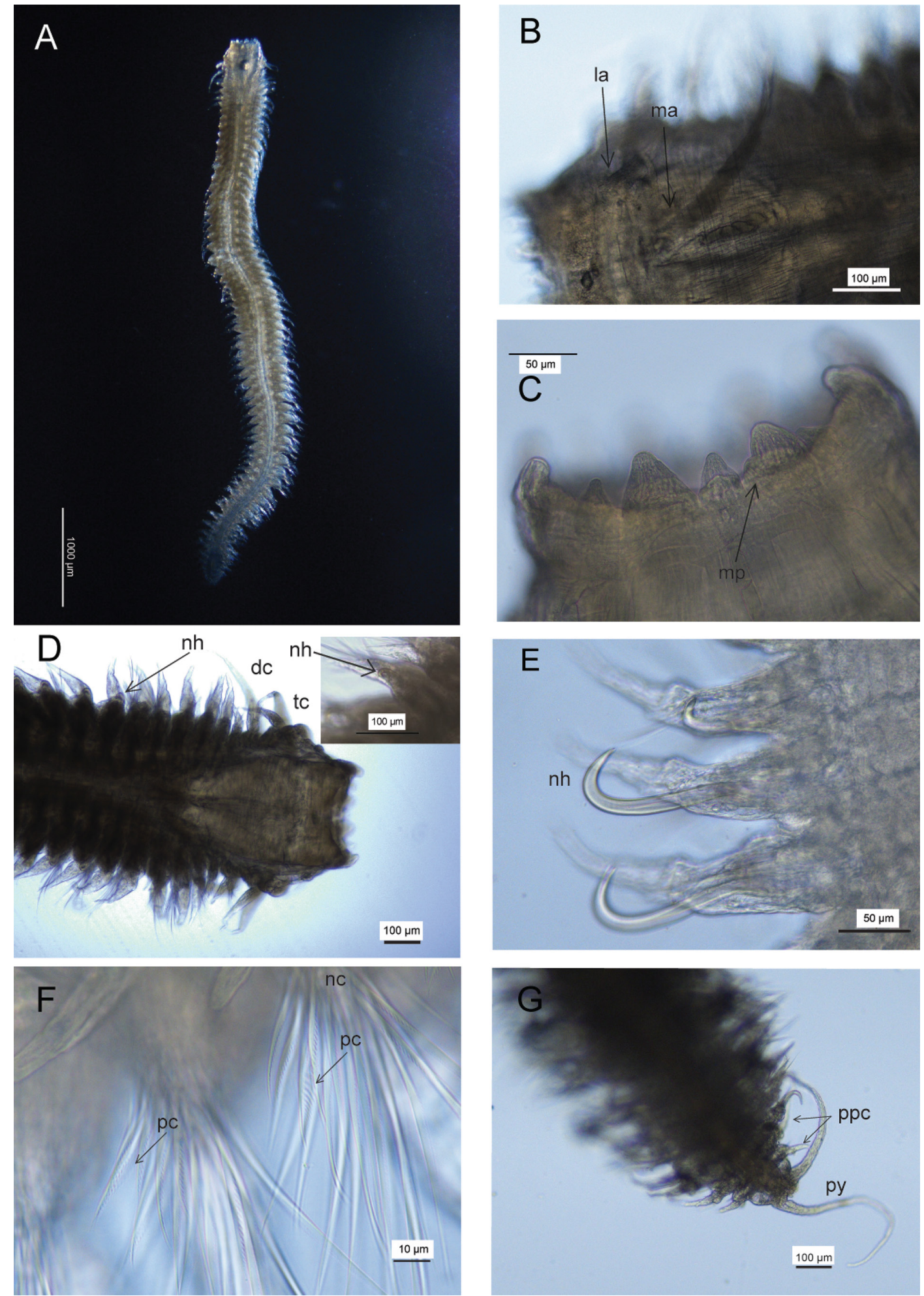

Fig. 3. Sigambra sundarbanensis sp. nov., holotype. A. Dorsal view (PUZ 501). B. Anterior end (dorsal view), lateral antenna (la) and median antenna (ma) in dorsal view. C. Pharynx, 7 among 14 marginal papillae (mp), dorsal view. D. First appearance of notopodial hook (nh, inset) from chaetiger 8, dorsal cirri $(\mathrm{dc})$ longest at chaetiger 1 ; tc $=$ tentacular cirri. E. Median chaetigers, continuous notopodial hooks (nh). F. Neuropodial chaeta (nc) with four short pectinates (pc). G. Pygidium (py) with last 2 prepygidial chaetigers (ppc) without hook. 


\begin{tabular}{|c|c|c|c|c|c|c|c|c|c|c|c|}
\hline 9 & 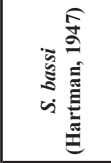 & 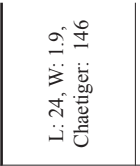 & 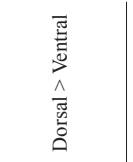 & & \pm & 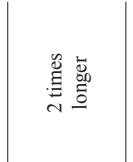 & a & 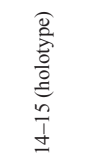 & 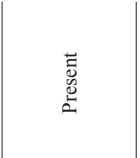 & 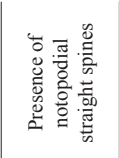 & 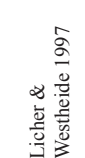 \\
\hline$\simeq$ & 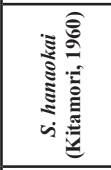 & 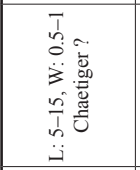 & 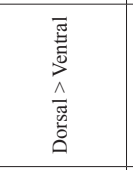 & $\begin{array}{l}\text { 咅 } \\
\text { 宸 }\end{array}$ & \pm & 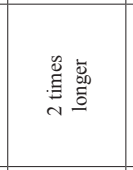 & 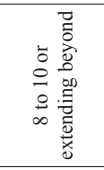 & $\stackrel{\infty}{\sim}$ & 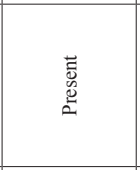 & & 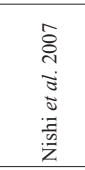 \\
\hline$=$ & 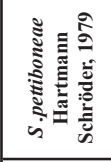 & 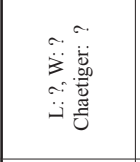 & 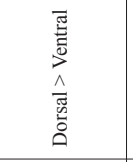 & $\begin{array}{l}\text { 言 } \\
\stackrel{0}{8}\end{array}$ & $=$ & 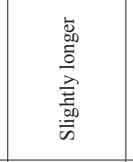 & 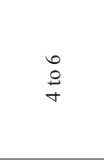 & $\stackrel{\circ}{i}$ & 1 & & 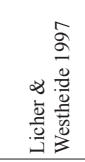 \\
\hline 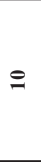 & 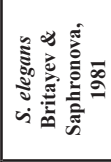 & 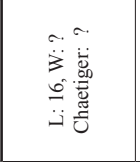 & 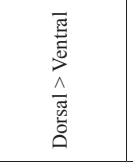 & $\begin{array}{l}\text { 咅 } \\
\text { 商 }\end{array}$ & $=$ & 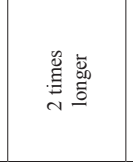 & 1 & $\stackrel{\infty}{\underline{I}}$ & I & & 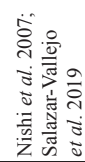 \\
\hline$a$ & 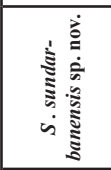 & 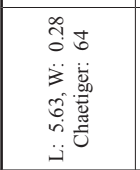 & 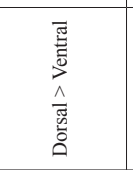 & 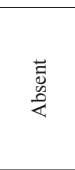 & \pm & 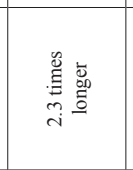 & $\underset{\substack{+0}}{+}$ & $\infty$ & 㠓 & 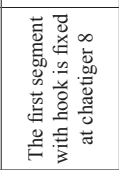 & 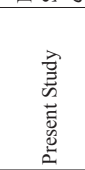 \\
\hline$\infty$ & 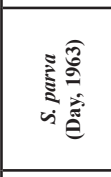 & 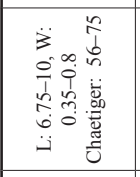 & 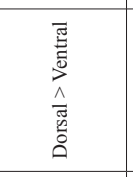 & $\begin{array}{l}\text { 言 } \\
\stackrel{0}{\gtrless}\end{array}$ & \pm & 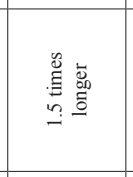 & 1 & in & 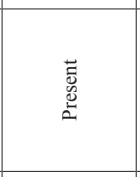 & 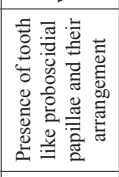 & 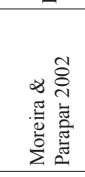 \\
\hline$r$ & 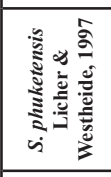 & 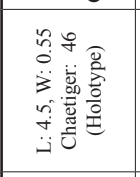 & 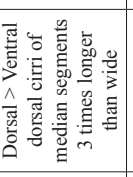 & 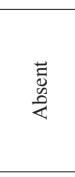 & \pm & 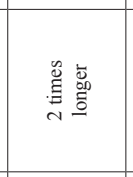 & $\stackrel{\overbrace{}}{\varrho}$ & $m$ & 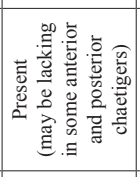 & & 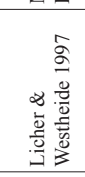 \\
\hline 0 & 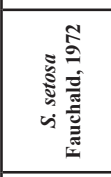 & 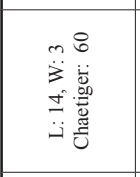 & 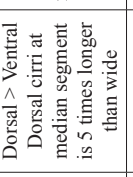 & $\begin{array}{l}\text { 言 } \\
\text { 岁 }\end{array}$ & 1 & 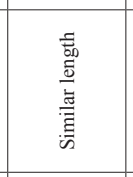 & 1 & \pm & 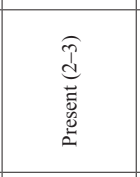 & 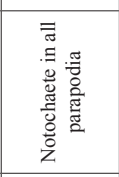 & 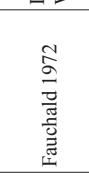 \\
\hline in & 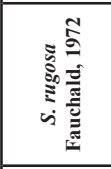 & 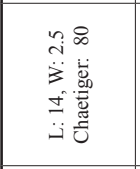 & 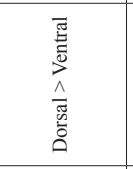 & $\begin{array}{l}\text { 言 } \\
\stackrel{0}{8}\end{array}$ & I & $\begin{array}{l}\text { 縍 } \\
\text { 离 }\end{array}$ & - & $\begin{array}{l}\stackrel{8}{0} \\
\dot{q}\end{array}$ & 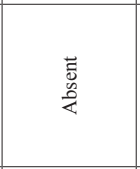 & & 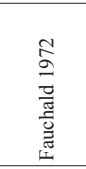 \\
\hline+ & 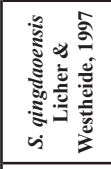 & 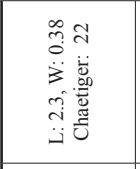 & 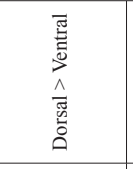 & $\begin{array}{l}\text { 言 } \\
\stackrel{0}{4}\end{array}$ & $\infty$ & 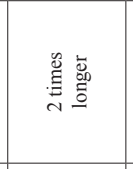 & m & p & $\begin{array}{l}\text { 言 } \\
\text { 总 }\end{array}$ & & 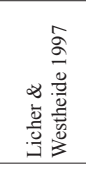 \\
\hline$m$ & 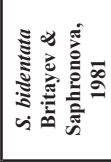 & I & 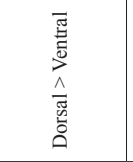 & $\begin{array}{l}\text { 言 } \\
\text { 商 }\end{array}$ & $\infty$ & 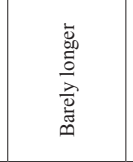 & 1 & m & 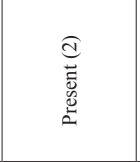 & 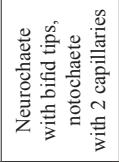 & 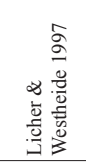 \\
\hline$\sim$ & 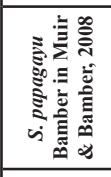 & 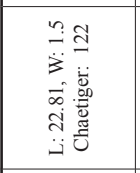 & 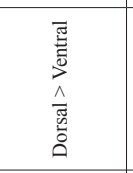 & 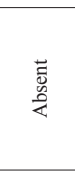 & $\infty$ & 总总 & in & $m$ & 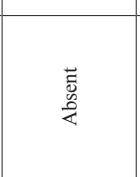 & 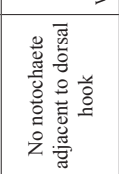 & 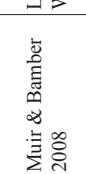 \\
\hline- & 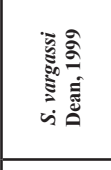 & 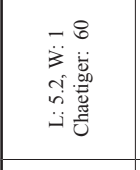 & 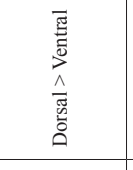 & 咅 & $\infty$ & 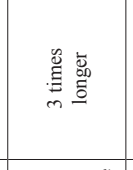 & $r$ & I & 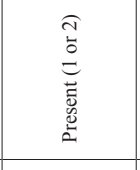 & 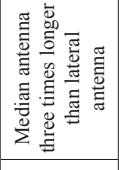 & 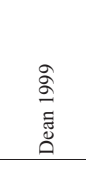 \\
\hline & 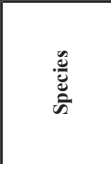 & 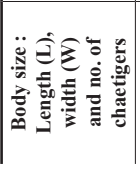 & 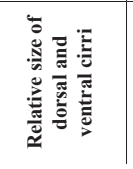 & 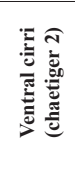 & 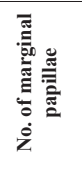 & 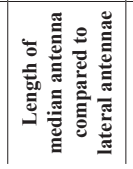 & 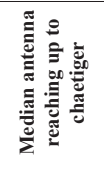 & 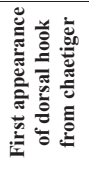 & 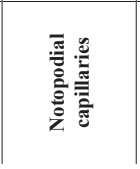 & 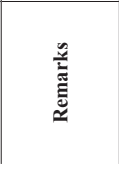 & 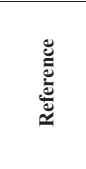 \\
\hline
\end{tabular}




\begin{tabular}{|c|c|c|c|c|c|c|c|c|c|c|c|}
\hline$\stackrel{\&}{2}$ & 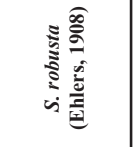 & 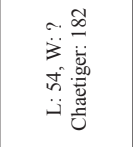 & 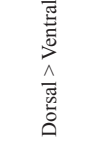 & 言 & $\begin{array}{l}\stackrel{0}{\overline{0}} \\
\stackrel{0}{n}\end{array}$ & & & 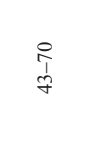 & 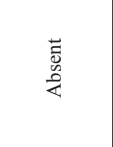 & 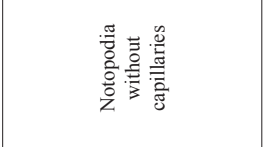 & 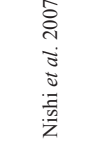 \\
\hline 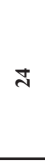 & 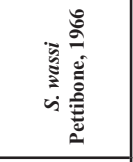 & 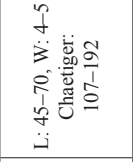 & 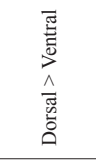 & 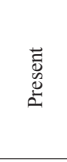 & $\infty$ & 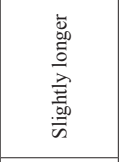 & & $\hat{\lambda}$ & 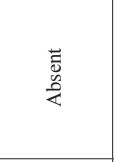 & & 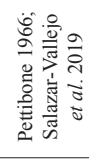 \\
\hline$\approx$ & 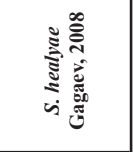 & 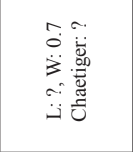 & 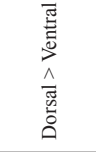 & 言 & $\infty$ & 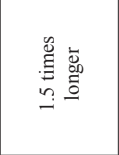 & .. & + & 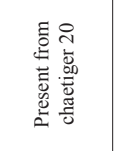 & 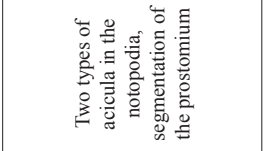 & 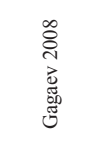 \\
\hline ส & 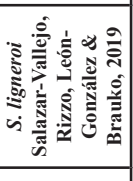 & 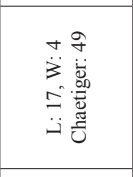 & 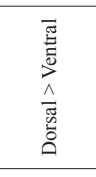 & 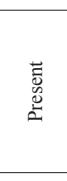 & $\infty$ & $\frac{8}{3}$ & $\stackrel{\widetilde{\Omega}}{\cong}$ & $\begin{array}{l}\text { đ్ } \\
\text { d్ }\end{array}$ & 厗 & 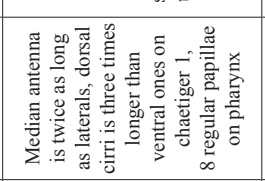 & 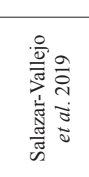 \\
\hline $\bar{\pi}$ & 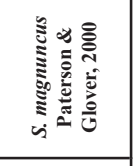 & 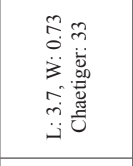 & 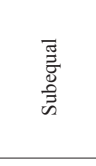 & 喜 & $\infty$ & 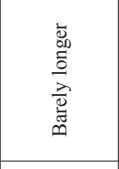 & $\underset{\substack{d \\
m}}{+}$ & $m$ & 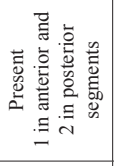 & & 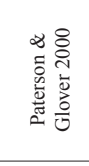 \\
\hline ते & 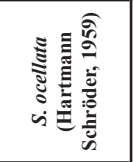 & 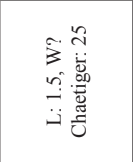 & $\begin{array}{l}\text { 受 } \\
\frac{\bar{g}}{\bar{n}}\end{array}$ & 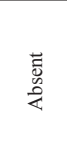 & $\infty$ & 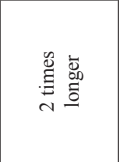 & 1 & b & 1 & 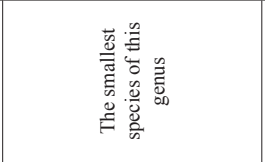 & 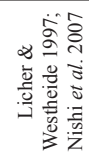 \\
\hline 2 & 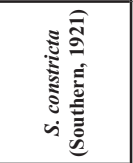 & 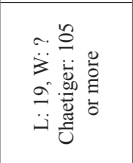 & 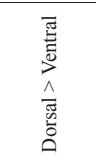 & 喜 & \pm & 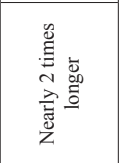 & $\stackrel{\tilde{\Omega}}{\stackrel{2}{2}}$ & 呆 & 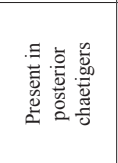 & 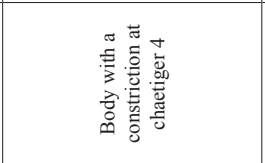 & 吾 \\
\hline$\stackrel{\infty}{\underline{\prime}}$ & 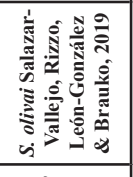 & 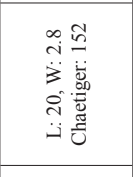 & 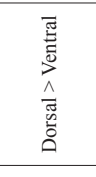 & 喜 & $\cong$ & $\frac{\mathscr{g}}{\vec{E}}$ & $\stackrel{3}{\stackrel{0}{i}}$ & $\stackrel{\infty}{\infty}$ & $\begin{array}{l}\text { 蒿 } \\
\text { 岁 }\end{array}$ & 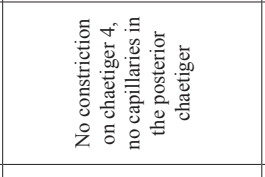 & 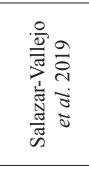 \\
\hline$=$ & 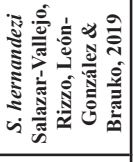 & 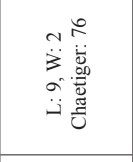 & 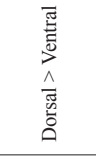 & 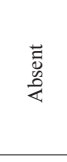 & \pm & $\frac{8}{E}$ & $\stackrel{3}{\stackrel{0}{i}}$ & $\stackrel{\sim}{\infty}$ & $\begin{array}{l}\text { 产 } \\
\frac{g_{0}}{4}\end{array}$ & & 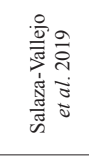 \\
\hline 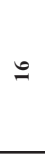 & 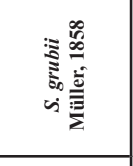 & 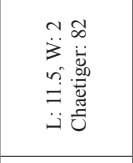 & 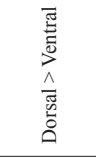 & 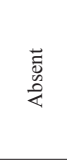 & \pm & $\begin{array}{l}\text { 总 } \\
\stackrel{5}{5}\end{array}$ & N & \& & 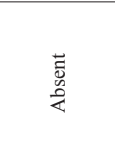 & 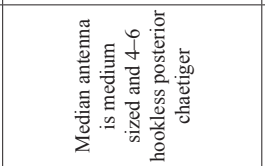 & 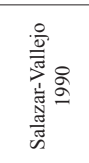 \\
\hline$\because$ & 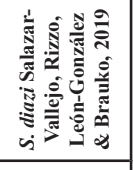 & 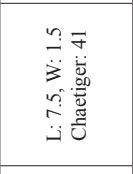 & 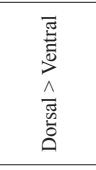 & 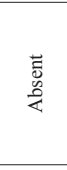 & $\begin{array}{l}\stackrel{0}{d} \\
\underline{a}\end{array}$ & 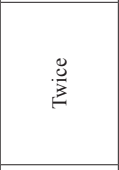 & $\hat{o}$ & q & $\begin{array}{l}\text { 言 } \\
\text { 樆 }\end{array}$ & 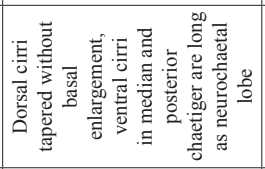 & 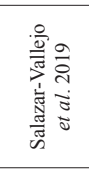 \\
\hline \pm & 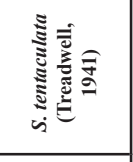 & 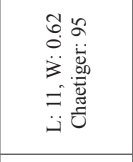 & 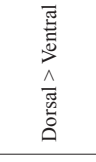 & $\begin{array}{l}\overline{\bar{y}} \\
\overrightarrow{8}\end{array}$ & \pm & 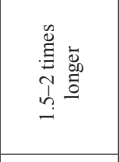 & & i & $\begin{array}{l}\text { 言 } \\
\text { 总 }\end{array}$ & 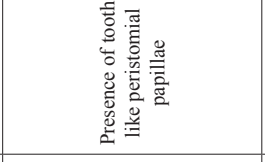 & 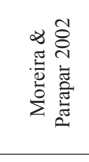 \\
\hline & 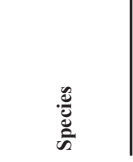 & 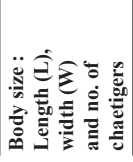 & 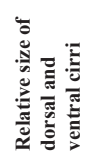 & 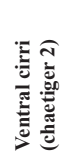 & 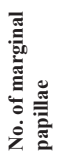 & 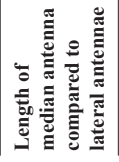 & 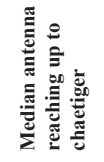 & 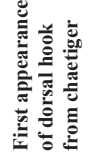 & 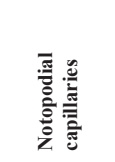 & 竧 & 产 \\
\hline
\end{tabular}


the posterior parapodial segments, neuropodia with 1-2 pectinate chaetae, but the number of hookless chaetigers is not mentioned in the literature (Day 1963; Moreira \& Parapar 2002).

\section{Distribution}

Sigambra sundarbanensis sp. nov. is only known from the rivers Matla and Thakuran of the Indian Sundarbans.
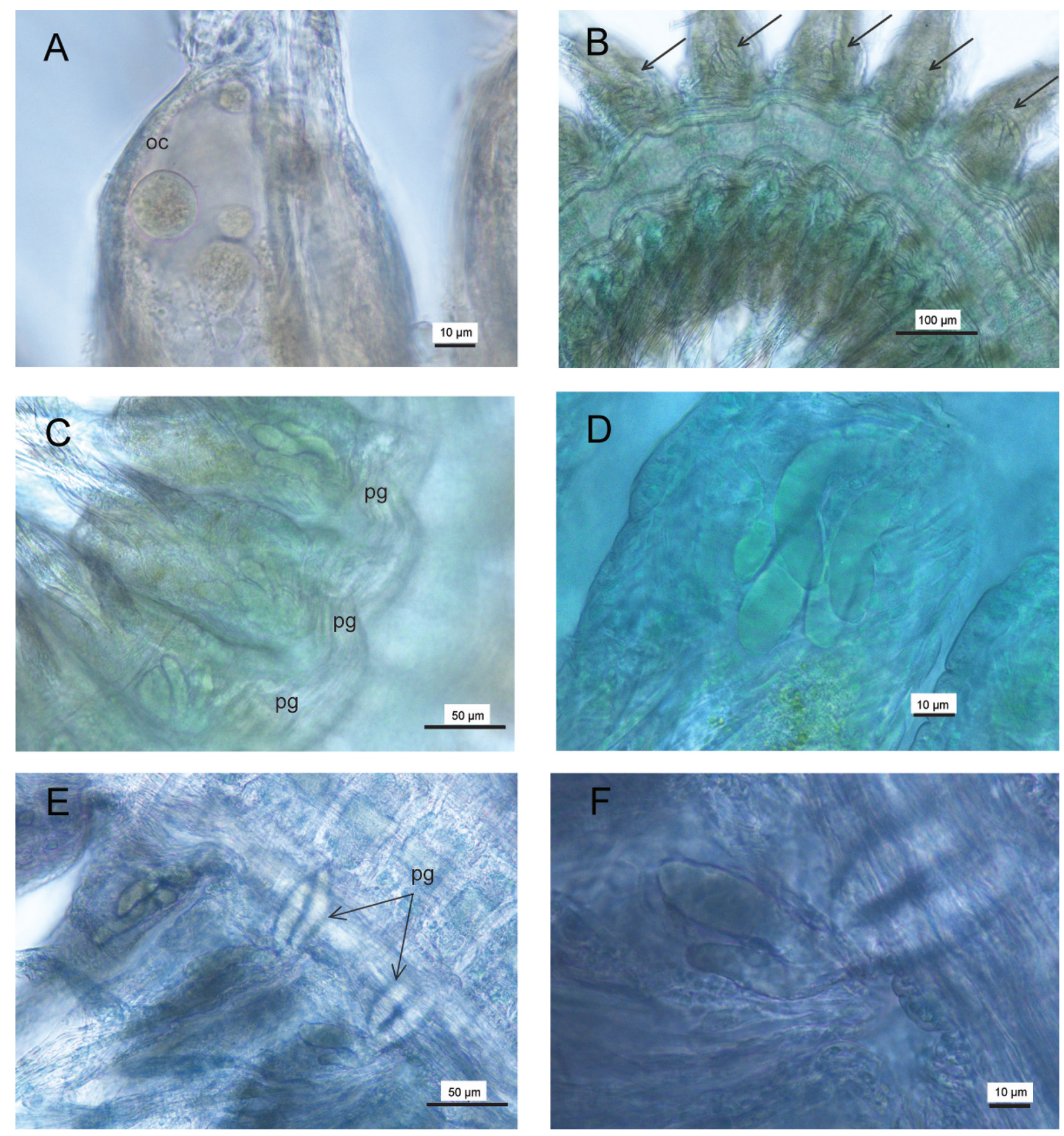

Fig. 4. Sigambra sundarbanensis sp. nov. A. Paratype (PUZ 503), oocytes inside parapodial spaces. B. Paratype (PUZ 506), arrows showing parapodial glands. C. Chaetigers 47-49 with parapodial glands (pg). D. Parapodial gland in $100 \times$ magnification. E-F. Paratype (PUZ 515), parapodial glands in chaetiger 12-13 invaded into coelomic space. 


\section{Ecology}

All specimens of this new species were found in mangrove habitats with silty sand sediments, in depths of 11 to $26 \mathrm{~m}$. Mature specimens, with developed oocytes, were recorded in August and December 2019 from Thakuran River. Among all the abiotic factors, salinity plays a pivotal role in ecology and
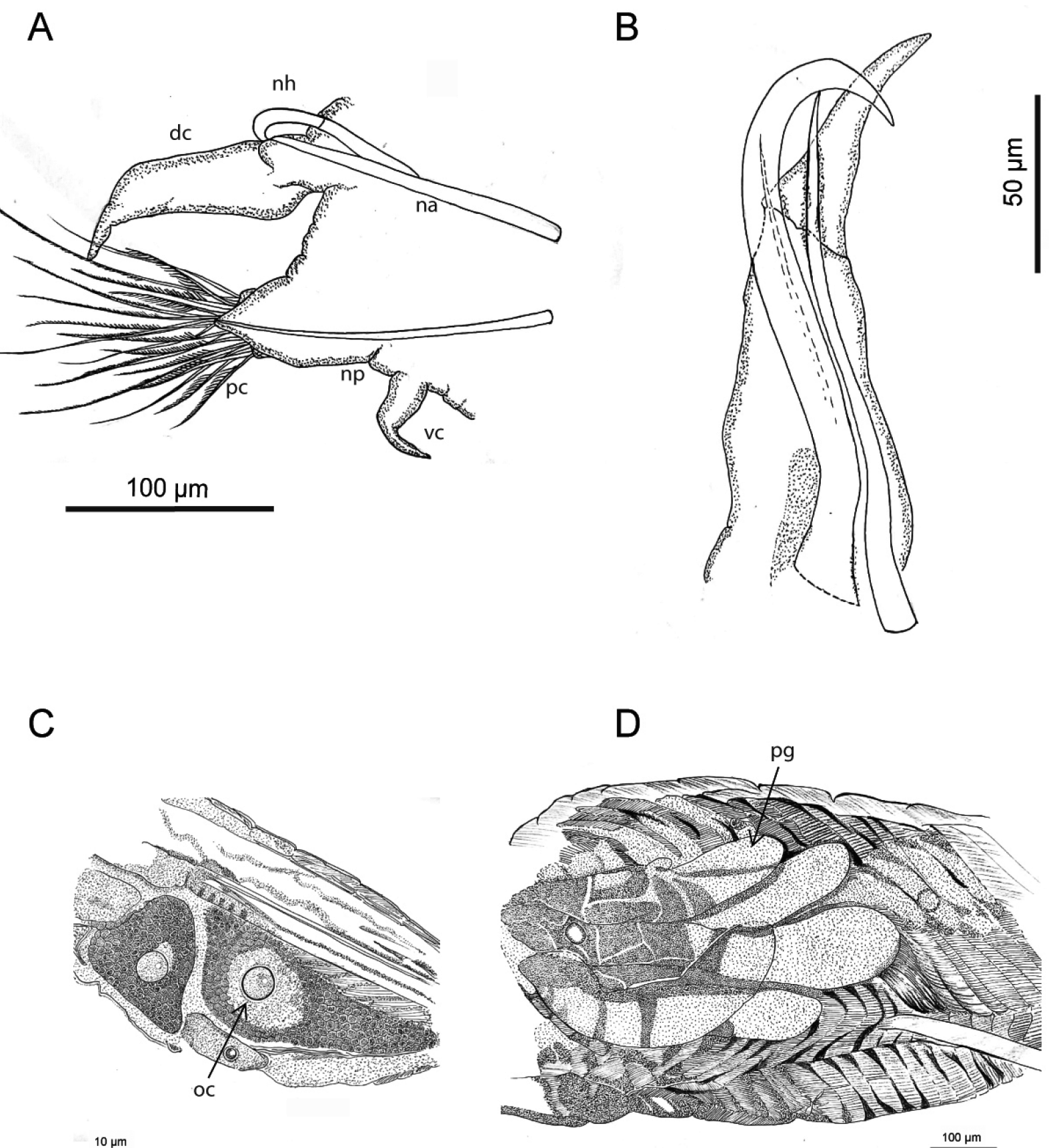

$10 \mu \mathrm{m}$

Fig. 5. Sigambra sundarbanensis sp. nov. A. Holotype (PUZ 501), parapodia with notopodial hook and neurochaetae. B. Paratype (PUZ 507), notopodium with hook, acicula and cirrus. C. Paratype (PUZ 514), oocytes inside parapodia. D. Holotype (PUZ 501), a bunch of large gland cells in parapodium. Abbreviations: $\mathrm{dc}=$ dorsal cirrus; $\mathrm{vc}=$ ventral cirrus; $\mathrm{np}=$ neuropodium; $\mathrm{pc}=$ pectinate chaeta; $\mathrm{na}=$ notoacicula; $\mathrm{nh}=$ notopodial hook; $\mathrm{oc}=$ oocyte; $\mathrm{pg}=$ parapodial gland. 
distribution of species across the globe, as this acts as a physiological barrier for both stenohaline and euryhaline species. Sigambra parva was recorded from Cape Province, South Africa (Day 1963) and the Mediterranean coast of Spain (Moreira \& Parapar 2002), where the water salinity remains higher than $30 \%$, whereas the localities of S. sundarbanensis sp. nov. had a salinity of $17-23.42 \%$. Additionally, S. parva had a comparatively higher range of depth variation from 2 to 97 meters (Day 1963; Moreira \& Parapar 2002).

\section{Key to species of Sigambra Müller, 1858}

(modified after Salazar-Vallejo et al. 2019)

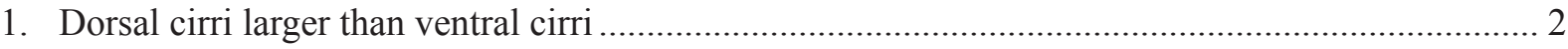

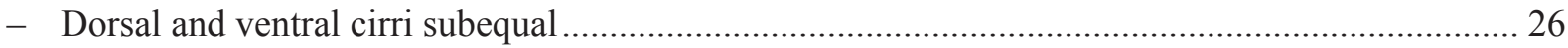

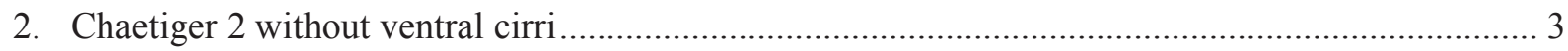

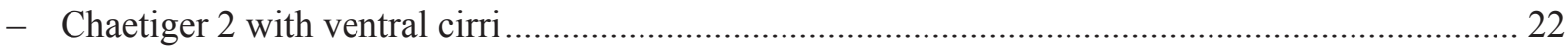

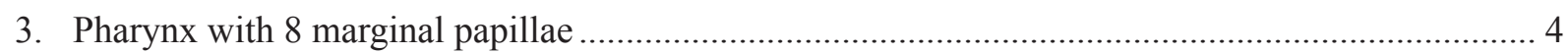

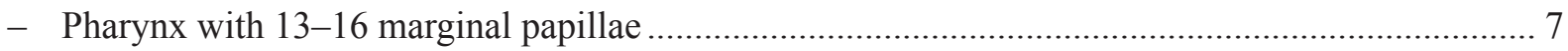

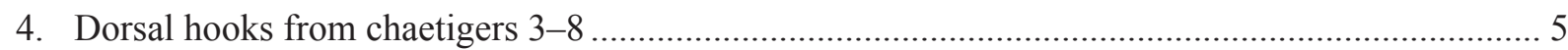

- Dorsal hooks from chaetigers 15-17 (5-18 mm long; up to chaetiger 30?); median and posterior notopodia with 1 capillary; median antenna long, reaching chaetiger 7 S. vargasi Dean, 1999

5. Median and posterior notopodia with capillaries.

- Notopodia without capillaries; all neurochaetae with tips entire; median antennae reaching chaetiger 5 (17-23 mm long) S. papagayu Bamber in Muir \& Bamber, 2008

6. Some neurochaetae with bifid tips; median antenna barely longer than laterals, reaching chaetiger 2; median and posterior notopodia with 2 capillaries

S. bidentata Britayev \& Saphronova, 1981

- All neurochaetae with entire tip; median antenna markedly longer than laterals, reaching chaetiger 3; median and posterior notopodia with 1 capillary ....... S. qingdaoensis Licher \& Westheide, 1997

7. Dorsal hooks from anterior chaetigers (4-18)

- Dorsal hooks from median chaetigers (30-40); median antenna twice as long as lateral ones or longer

- Dorsal hooks from posterior chaetigers (42-66), or beyond that (14 mm long); median antenna as long as lateral ones, barely reaching chaetiger 1

S. rugosa Fauchald, 1972

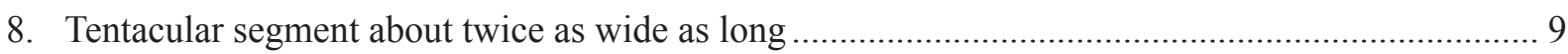

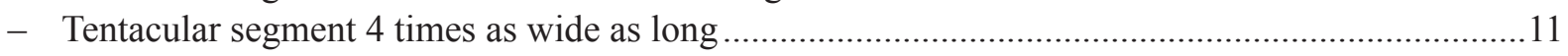

9. Tentacular segment with anterior margin with rounded projected lobes, external to lateral antennae.

- Tentacular segment with anterior margin smooth, without projected lateral lobes; dorsal hooks from chaetiger 3-4 (14 mm long).

10. Antennae of similar length; dorsal cirri of median segments 5 times as long as wide

- Median antenna almost twice as long as lateral ones; dorsal cirri of median segments 3 times as long as wide 
11. Tentacular segment with rounded projected lobes in anterior margin; median antenna slightly longer than lateral ones, reaching chaetigers $3-4$

- Tentacular segment with anterior margin smooth, without rounded projected lobes 13

12. Dorsal hooks from chaetigers $4-5$ (6-12 $\mathrm{mm}$ long)...... S. parva (Day, 1963)

- Dorsal hooks from chaetiger 8 (2-9 mm long). S. sundarbanensis sp. nov.

13. Median and posterior notopodia with capillaries. 14

- Notopodia without capillaries. 20

14. Median antenna short, reaching up to chaetigers 3-4. 15

- Median antenna medium-sized, reaching chaetigers 5-7. 16

- Median antenna long, reaching chaetigers 9-12; dorsal hooks of chaetigers 11-15 (40 mm long); lateral antennae without lateral depressions S. bassi sensu Blake, 1994

15. Dorsal hooks from chaetiger 4 (15 mm long); median antenna slightly longer than lateral ones; first dorsal cirri slightly longer than dorsal tentacular ones S. tentaculata sensu Blake, 1994

- Dorsal hooks from chaetiger 12-18 (16 mm long); median antenna twice as long as lateral ones; first dorsal cirri markedly longer than dorsal tentacular ones....S. elegans Britayev \& Saphronova, 1981

16. Median antenna slightly longer than lateral ones, reaching chaetigers 4-6; dorsal hooks from chaetigers 7-10 (5.5 mm long) S. pettiboneae Hartmann-Schröder, 1979

- Median antenna twice as long as lateral ones. 17

17. Median antenna reaching chaetigers $7-8$ 18

- Median antenna reaching up to chaetigers 5-6; dorsal hooks from chaetigers 4-5 19

18. Dorsal hooks from chaetigers 3-9 (5-20 mm long) S. hanaokai (Kitamori, 1960)

- Dorsal hooks from chaetigers 12-18 (24 mm long)..... S. bassi (Hartman, 1947)

19. All parapodia with ventral cirri shorter than neuropodial lobes; dorsal cirri basally wider

S. tentaculata (Treadwell, 1941)

- Median and posterior parapodia with ventral cirri long, reaching tip of neuropodial lobes; dorsal cirri tapered, not wider basally

S. diazi Salazar-Vallejo et al., 2019

20. Median antenna medium-sized, reaching chaetigers 3-4; posterior region with 4-6 hookless chaetigers; body papillae large in proportion

S. grubii Müller, 1858

- Median antenna short, reaching chaetigers 2-3; posterior region with 2 hookless chaetigers; body papillae small S. hernandezi Salazar-Vallejo et al., 2019

21. Median antenna slightly longer than laterals; body without a constriction on chaetiger 4; dorsal hooks from chaetigers 30-39 (15-24 mm long); posterior chaetigers without capillary notochaetae

S. olivai Salazar-Vallejo et al., 2019

- Median antenna twice as long as laterals; body with a constriction on chaetiger 4; dorsal hooks from chaetiger 30-40 (16-24 mm long); posterior chaetigers with a single capillary notochaetae.

S. constricta (Southern, 1921)

22. Pharynx with 8 marginal papillae

- Pharynx with 14-16 marginal papillae 
23. Median antenna slightly longer than lateral ones

- Median antenna twice as long as lateral ones; dorsal hooks from chaetigers 26-28.

S. ligneroi Salazar-Vallejo et al., 2019

24. Dorsal hooks from chaetiger 4 (0.7 mm wide), tentacular segment as long as wide

S. healyae Gagaev, 2008

- Dorsal hooks from chaetigers 23-30 (45-70 mm long); tentacular segment 4-5 times as wide as long.

S. wassi Pettibone, 1966

25. Dorsal hooks from chaetiger 7, with accessory capillaries; dorsal cirri progressively longer from chaetiger 2 .

Sigambra sp. Imajima, 2001

- Dorsal hooks from chaetigers 43-70, without capillary chaetae; dorsal cirri of chaetiger 2 smaller than those in following chaetigers

S. robusta (Ehlers, 1908)

26. Dorsal hooks from chaetiger 6 (1.5 $\mathrm{mm}$ long)

S. ocellata (Hartmann-Schröder, 1959)

- Dorsal hooks from chaetiger 3 (3.7 mm long). S. magnuncus (Paterson \& Glover, 2000)

*As per Salazar-Vallejo et al. (2019), this is not completely identified and based upon some specimens which have not been described yet, but are distinct and deserve to be described.

\section{Discussion}

In general, there are two patterns regarding positional variation of notopodial hooks in Sigambra. It either starts from a specific chaetiger (1-3 chaetiger variation) or shows variation. The position of the notopodial hook in $S$. sundarbanensis sp. nov. follows the first pattern, whereas most of its congeners show variations. The general variability in the first appearance of the hooks and the number of hookless chaetigers in pre-pygidial segments can limit its taxonomic position (Licher \& Westheide 1997).

Based on literature, Sigambra sundarbanensis sp. nov. is the first reported species of this genus with parapodial glandular structures. According to the methyl green stained images, these glands resemble the chromophile glands found in the neuropodial pinnae among the members of Tomopteridae (Grube, 1850) (e.g., Tomopteris helgolandica Greeff, 1879) which are pelagic in nature and involved in light production (Gouveneaux et al. 2017). For the taxonomic classification of tomopterids, Fauvel (1923) mentioned these glands and their affinity towards nuclear dyes like haematoxylin. Later on, a detailed histochemical examination on these light-producing parapodial glands by Gouveneaux (2016) confirmed their similar staining property. However, no such parapodial structures have so far been reported for any species of Sigambra in particular or pilargids in general. Even though the histochemistry of this gland is unknown, methyl green staining images (Fig. 4B-F) depict a visual resemblance with the image in Gouveneaux et al. (2017: fig. 2f). Moreover, the first appearance of these glands from the anterior parapodia along with their gradual enlargement can be similar to what is shown in specimens of Enapteris euchaeta Chun, 1888 or Tomopteris elegans Chun, 1888. Their chromophile glands are very conspicuous and situated inferiorly from segment 4, although in Tomopteris apsteini Rosa, 1908 they are visible from the third pair of parapodia, progressively larger, becoming enormous globes hanging under ventral rami (Støp-Bowitz 1948; Böggemann 2009). However, without having a detailed knowledge on its histochemistry and function, the observed structure in our study should not be further named; rather, it would be more appropriate referring them as parapodial glands.

Sigambra sundarbanensis sp. nov. is described from a mangrove dominated estuary of the Indian Sundarbans. The species has been described with morphological features along with the environmental factors in different seasons from the rivers Matla and Thakuran, Sundarbans. This report contributes to the polychaete checklist of Indian waters. It also includes the first documentation of some parapodial 
glands in Sigambra. Moreover, it also delivers a global map of the type localities of all the accepted species of Sigambra.

\section{Acknowledgements}

The authors wish to express their gratitude to The Vice Chancellor, Presidency University for providing facilities and encouragement to carry out the above research work. The authors thank anonymous reviewers for their constructive comments for improving the overall quality of manuscript. The authors are thankful to Dr Meenakshi Chatterjee, Basanti Devi College, Kolkata for her ever willing help and cooperation during the study. We also thank Dr Dhiraj Narale for extracting the world map for Fig. 2. This work was supported by a grant awarded to S.M. from the Ministry of Earth Sciences (MoES/36/OOIS/Extra/24/2013 dated 11 Apr. 2016). Thanks are also due to the Director, Sundarbans Biosphere Reserve and the Principal Chief Conservator of Forest, Directorate of Forest, Government of West Bengal for their continuous support and encouragement (permission letters memo no.3100/ WL/4R-6/2018 dt. 05.09.2018 and C/28011/24/2019 dated 02.08.2019). The authors would also like to express their appreciation to the members of the Marine Ecology Laboratory for excellent team work.

\section{Funding information}

This work is supported by a grant awarded to S.M. from Ministry of Earth Sciences (MoES/36/OOIS/ Extra/24/2013 dated 11 Apr. 2016) and FRPDF grant of Presidency University.

\section{Compliance with ethical standards}

\section{Conflict of interest}

The authors declare that they have no conflict of interest.

\section{Ethical approval}

No animal testing was performed during this study.

\section{Sampling and field studies}

All necessary permits for field studies and sampling have been obtained by the authors from the competent authorities and are mentioned in the acknowledgements.

\section{Data availability statement}

All data generated or analyzed during this study are included in this published article.

\section{References}

Achari G.P.K. 1975. Studies on new or little known polychaetes from the Indian Seas, 4. On a new record of Sigambra tentaculata (Treadwell) (Pilargidae), from the southwest coast of India along with observations on its early larval stages. Journal of the Marine Biological Association of India 17 (2): $238-241$.

Böggemann M. 2009. Polychaetes (Annelida) of the abyssal SE Atlantic. Organisms, Diversity \& Evolution 9: 251-428.

Buchanan J.B. 1984. Sediment analysis. In: Holme N.A. \& McIntyre A.D. (eds) Methods for the Study of Marine Benthos: 41-65. Blackwell Scientific Publications, Oxford.

Dahlgren T.G., Lundberg J., Pleijel F. \& Sundber P. 2000. Morphological and molecular evidence of the phylogeny of nereidiform polychaetes (Annelida). Journal of Zoological Systematics and Evolutionary Research 38: 249-253. https://doi.org/10.1046/j.1439-0469.2000.384150.x 
Day J.H. 1963. The polychaete fauna of South Africa. 8. New species and records from grab samples and dredgings. Bulletin of the British Museum (Natural History), Zoology 10 (7): 381-445.

https://doi.org/10.5962/bhl.part.20530

Day J.H. 1967. A monograph on the polychaetes of Southern Africa. Part 1: Errantia, Part 2: Sedentaria. British Museum (Natural History), London, Publications 656: 1-878.

https://doi.org/10.5962/bhl.title.8596

Dean H.K. 1998. The Pilargidae (Annelida: Polychaeta) of the Pacific coast of Costa Rica. Revista de Biologia Tropical 46 (6): 47-62.

Available from https://revistas.ucr.ac.cr/index.php/rbt/article/view/29633 [accessed 15 Mar. 2021].

El Wakeel S.K. \& Riley J.P. 1957. The determination of organic carbon in marine muds. ICES Journal of Marine Science 22 (2): 180-183. https://doi.org/10.1093/icesjms/22.2.180

Fauchald K. 1972. Benthic polychaetous annelids from deep waters off western Mexico and adjacent areas in the Eastern Pacific Ocean. Allan Hancock Monograph in Marine Biology 7: 1-575.

Available from https://repository.si.edu/handle/10088/6207 [accessed 15 Mar. 2021].

Fauvel P. 1923. Polychètes errantes. Faune de France 5: 217-225.

Gagaev S.Y. 2008. Sigambra healyae n. sp., a new species of polychaete (Polychaeta: Pilargidae) from the Canadian Basin of the Arctic Ocean. Russian Journal of Marine Biology 34 (1): 73-75.

https://doi.org/10.1134/S1063074008010100

Gouveneaux A. 2016. Bioluminescence of Tomopteridae species (Annelida): multidisciplinary approach. $\mathrm{PhD}$ Thesis, Université Catholique de Louvain, Belgium.

Available from http://hdl.handle.net/2078.1/176773 [accessed 15 Mar. 2021].

Gouveneaux A., Flood P.R., Erichsen E.S., Olsson C., Lindström J. \& Mallefet J. 2017. Morphology and fluorescence of the parapodial light glands in Tomopteris helgolandica and allies (Phyllodocida: Tomopteridae). Zoologischer Anzeiger 268: 112-125. https://doi.org/10.1016/j.jcz.2016.08.002

Grasshoff K., Kremling K. \& Ehrhardt M. 1999. Methods of Seawater Analysis (3 ${ }^{\text {rd }}$ ed.). Verlag Chemie, Weinheim, Germany. https://doi.org/10.1002/9783527613984

Hartman O. 1947. Polychaetous Annelids. Part 8. Pilargidae. Allan Hancock Pacific Expeditions 10: 483-523.

Jumars P.A., Dorgan K.M. \& Lindsay S.M. 2015. Diet of worms emended: an update of polychaete feeding guilds. Annual Review of Marine Science 7 (1): 497-520.

https://doi.org/10.1146/annurev-marine-010814-020007

Licher F. \& Westheide W. 1994. The phylogenetic position of the Pilargidae with a cladistic analysis of the taxon - facts and ideas. In: Dauvin J.C., Laubier L. \& Reish D.J. (eds) Actes de la $4^{\mathrm{eme}}$ Conférence internationale des Polychètes. Mémoires du Museum national d'histoire naturelle 162: 223-235.

Licher F. \& Westheide W. 1997. Review of the genus Sigambra (Polychaeta: Hesionidae), redescription of S. bassi (Hartman 1947) and descriptions of two new species from Thailand and China. Steenstrupia 23: $1-20$.

Mandal S. \& Deb S. 2018. Ancistrosyllis matlaensis n. sp. (Polychaeta: Pilargidae) from the Sundarban Estuarine System, India. Zootaxa 4531 (3): 419-429. https://doi.org/10.11646/zootaxa.4531.3.6

Manna S., Chaudhuri K., Bhattacharyya S. \& Bhattacharyya M. 2010. Dynamics of Sundarban estuarine ecosystem: eutrophication induced threat to mangroves. Saline System 6 (8): 1-16.

https://doi.org/10.1186/1746-1448-6-8 
Misra A. 1995. Polychaetes. In: Director, Zoological Survey of India (ed.) Hugli Matla Estuary. Zoological Survey of India, Calcutta, Estuarine Ecosystem Series 2: 93-155.

Moreira J. \& Parapar J. 2002. Redescription of Sigambra tentaculata and re-establishment of S. parva (Polychaeta, Pilargidae) based upon type material. Cahiers de Biologie marine 43: 99-109.

Muir A.I. \& Bamber R.N. 2008. New polychaete (Annelida) records and a new species from Hong Kong: the families Polynoidae, Sigalionidae, Chrysopetalidae, Pilargiidae, Nereididae, Opheliidae, Ampharetidae and Terebellidae. Journal of Natural History 42 (9-12): 797-814.

https://doi.org/10.1080/00222930701850455

Müller F. 1858. Einiges über die Annelidenfauna der Insel Santa Catharina an der brasilianischen Küste. Archiv für Naturgeschichte, Berlin 24: 211-220.

Available from https://biodiversitylibrary.org/page/7460059 [accessed 15 Mar. 2021].

Nishi E., Tanaka K., Fujioka Y. \& Sato, M. 2007. Reinstatement of Sigambra hanaokai (Kitamori, 1960) (Polychaeta, Pilargidae), with an overview of the literature on the genus. Zootaxa 1653 (1): 57-68. https://doi.org/10.11646/zootaxa.1653.1.4

Paterson G.L.J. \& Glover A.G. 2000. A new species of Sigambra (Polychaeta, Pilargidae) from the abyssal plains of the NE Atlantic. Bulletin of the Natural History Museum, London (Zoology) 66: 167170. Available from https://biodiversitylibrary.org/page/41005372 [accessed 15 Mar. 2021].

Pettibone M.H. 1966. Revision of the Pilargidae (Annelida: Polychaeta), including descriptions of new species, and redescription of the pelagic Podarmus ploa Chamberlin (Polynoidae). Proceedings of the United States National Museum 118: 155-207. https://doi.org/10.5479/si.00963801.118-3525.155

Pleijel F. \& Dahlgren T.G. 1998. Position and delineation of Chrysopetalidae and Hesionidae (Annelida, Polychaeta, Phyllodocida). Cladistics 14: 129-150. https://doi.org/10.1111/j.1096-0031.1998.tb00327.x

Raha A., Das S., Banerjee K. \& Mitra A. 2012. Climate change impacts on Indian Sunderbans: a time series analysis (1924-2008). Biodiversity Conservation 2: 1289-1307.

https://doi.org/10.1007/s10531-012-0260-z

Salazar-Vallejo S.I. 1986. Pilargidae (Annelida: Polychaeta) de México: lista de especies, nueva especie y biogeografía. Cahiers de Biologie marine 27: 193-209.

Salazar-Vallejo S.I. \& Rizzo A.E. 2009. Pilargidae de Saint-Joseph, 1899. In: de León-González J.A., Bastida-Zavala J.R., Carrera-Parra L.F., García-Garza M.E., Peña-Rivera A., Salazar-Vallejo S.I. \& Solís-Weiss V. (eds) Poliquetos (Annelida: Polychaeta) de México y América Tropical. Universidad Autónoma de Nuevo León, Monterrey.

Salazar-Vallejo S.I., Rizzo A.E., León-González J.Á. \& Brauko K.M. 2019. Four new Caribbean Sigambra species (Annelida, Pilargidae), and clarifications of three other Sigambra species. ZooKeys 893: 21-50. https://doi.org/10.3897/zookeys.893.39594

Southern R. 1921. Polychaeta of the Chilka Lake and also of fresh and brackish waters in other parts of India. Memoirs of Indian Museum, Calcutta 5: 563-659.

Available from https://biodiversitylibrary.org/page/36511332 [accessed 15 Mar. 2021].

Stanley D.J. \& Hait A.K. 2000. Holocene depositional patterns, neotectonics and Sundarban mangroves in the western Ganges-Brahmaputra delta. Journal of Coastal Research 16: 26-39.

Available from https://www.jstor.org/stable/4300009 [accessed 15 Mar. 2021].

Støp-Bowitz C. 1948. Polychaeta from the "Michael Sars" North Atlantic Deep-Sea Exp. 1910. Report on the Scientific Results of the Michael Sars North Atlantic Deep-Sea Expedition 8: 1-91. 
WoRMS Editorial Board. 2020. World Register of Marine Species.

Available from http://www.marinespecies.org [accessed 2 Oct. 2020]. https://doi.org/10.14284/170

Manuscript received: 2 October 2020

Manuscript accepted: 17 January 2021

Published on: 7 April 2021

Topic editor: Rudy C.A.M. Jocqué

Desk editor: Kristiaan Hoedemakers

Printed versions of all papers are also deposited in the libraries of the institutes that are members of the EJT consortium: Muséum national d'histoire naturelle, Paris, France; Meise Botanic Garden, Belgium; Royal Museum for Central Africa, Tervuren, Belgium; Royal Belgian Institute of Natural Sciences, Brussels, Belgium; Natural History Museum of Denmark, Copenhagen, Denmark; Naturalis Biodiversity Center, Leiden, the Netherlands; Museo Nacional de Ciencias Naturales-CSIC, Madrid, Spain; Real Jardín Botánico de Madrid CSIC, Spain; Zoological Research Museum Alexander Koenig, Bonn, Germany; National Museum, Prague, Czech Republic. 